\title{
Overexpression of miR-185 inhibits autophagy and apoptosis of dopaminergic neurons by regulating the AMPK/mTOR signaling pathway in Parkinson's disease
}

\author{
ZHI WEN ${ }^{1,2}$, JIE ZHANG $^{3}$, PENG TANG ${ }^{4}$, NING TU $^{2}$, KE WANG $^{2}$ and GUANGYAO WU ${ }^{2}$ \\ ${ }^{1}$ Department of Radiology, Renmin Hospital of Wuhan University, Wuhan, Hubei 430060; \\ Departments of ${ }^{2}$ Magnetic Resonance Imaging, ${ }^{3}$ Neurosurgery and ${ }^{4}$ Clinical Laboratory, \\ Zhongnan Hospital of Wuhan University, Wuhan, Hubei 430071, P.R. China
}

Received May 27, 2016; Accepted April 20, 2017

DOI: $10.3892 / \mathrm{mmr} .2017 .7897$

\begin{abstract}
Parkinson's disease (PD) is an age-associated neurodegenerative disorder characterized by the death of dopaminergic neurons in the substantia nigra pars compacta. Activation of 5'-adenosine monophosphate-activated protein kinase (AMPK) has been suggested to be associated with PD pathogenesis. The aim of the present study was to investigate the effects of the aberrant expression of microRNA-185 (miR-185) in PD. A 1-methyl-4-phenyl-1,2,3,6-tetrahydropyridine (MPTP)-induced in vitro model of PD was generated using the human SH-SY5Y dopaminergic neuroblastoma cell line, in order to examine the potential molecular mechanisms underlying the roles of miR-185 in PD. miR-185 expression was assessed in MPTP-treated SH-SY5Y cells using reverse transcription-quantitative polymerase chain reaction (RT-qPCR). In addition, MPTP-treated SH-SY5Y cells were transfected with a miR-185 mimic or scramble miRNA, and flow cytometry was used to evaluate the level of cellular apoptosis. The expression of autophagy markers, including Beclin 1, microtubule-associated protein light chain 3 (LC3) I and LC3II, as well as key molecules involved in the AMPK/mechanistic target of rapamycin (mTOR) signaling pathway, such as phosphorylated (p)-AMPK and p-mTOR, was examined using RT-qPCR and western blot analyses. In addition, SH-SY5Y cells were treated with the AMPK inhibitor, Compound $\mathrm{C}$, and its effects on cellular apoptosis were assessed. The results demonstrated that miR-185 was significantly downregulated in SH-SY5Y cells treated with MPTP at concentrations of $>100 \mu \mathrm{M}$ when compared with untreated controls. Following transfection with
\end{abstract}

Correspondence to: Professor Guangyao Wu, Department of Magnetic Resonance Imaging, Zhongnan Hospital of Wuhan University, 169 Donghu Road, Wuhan, Hubei 430071, P.R. China E-mail: wuguangy2002@163.com

Key words: Parkinson's disease, microRNA-185, apoptosis, autophagy, 5'-adenosine monophosphate-activated protein kinase/ mechanistic target of rapamycin pathway a miR-185 mimic, miR-185 expression in SH-SY5Y cells was significantly increased when compared with blank control cells. Notably, miR-185 overexpression was revealed to significantly reduce the MPTP-induced increase in cellular apoptosis. In addition, the expression levels of Beclin 1, LC3I/II, p-AMPK and p-mTOR were significantly upregulated in MPTP-treated SH-SY5Y cells; whereas miR-185 overexpression significantly downregulated the expression of these factors. Furthermore, miR-185 overexpression significantly suppressed apoptosis of SH-SY5Y cells treated with MPTP plus Compound C when compared with the Compound $\mathrm{C}$ group. In conclusion, the results of the present study suggest that overexpression of miR-185 may inhibit autophagy and apoptosis of dopaminergic cells in PD potentially via regulation of the AMPK/mTOR signaling pathway. Therefore, AMPK/mTOR-mediated autophagy and apoptotic signaling pathways may be potential novel therapeutic targets for the development of alternative strategies for the treatment of patients with PD.

\section{Introduction}

Parkinson's disease (PD) is an age-associated neurodegenerative disorder characterized by the death of dopaminergic (DAergic) neurons in the substantia nigra pars compacta $(1,2)$. It is the second most common neurodegenerative disease after Alzheimer's disease, always occurring in people over 60 years old (3). The principal manifestations of PD include a resting tremor, rigidity, hypokinesia and postural instability $(4,5)$. It is reported that PD can be caused by environmental risk factors and genetic factors (6-8). Despite major advances in the understanding of PD pathophysiology, no treatments are currently available to prevent disease progression (9). Additionally, the exact molecular mechanisms that initiate PD are still unclear (10). Therefore, elucidating the molecular mechanisms involved in the pathogenesis of PD is imperative for the development of novel effective therapeutic approaches for the treatment of patients with PD.

MicroRNAs (miRNAs) are small, endogenous, noncoding RNA molecules, which have exhibited potential as biomarkers or therapeutic targets for PD (11-13). Bioinformatics analysis has revealed that $\sim 200$ miRNAs, including miR-627, miR-634, 
miR-514, miR-563 and miR-613, are differentially expressed between PD and healthy tissue samples (14), suggesting that miRNAs may participate in PD pathogenesis and progression. In addition, upregulation of miR-124 has been demonstrated to regulate autophagy and apoptotic processes, and thus reduce the loss of DAergic neurons in a 1-methyl-4-phenyl-1,2,3,6-tetrahydropyridine (MPTP)-induced model of PD (15). Furthermore, miR-7 has been reported to possess neuroprotective properties, exerted through the inhibition of neuronal apoptosis in an in vitro model of PD (16). It is also reported that miR-7 can modulate neuroinflammation in the pathogenesis of PD through targeting Nod-like receptor protein 3 inflammasome (17). Therefore, it is hypothesized that miRNAs serve critical roles in PD via the regulation of autophagy and apoptotic pathways in DAergic neurons. Recently, miR-185 expression was reported to be downregulated in serum samples isolated from patients with PD compared with in healthy individuals (18). However, the roles of miR-185 in PD, as well as the molecular mechanisms underlying its involvement in the pathogenesis of the disease, have yet to be elucidated.

The present study investigated the expression of miR-185 in an MPTP-induced in vitro model of PD using the human SH-SY5Y DAergic neuroblastoma cell line. In addition, the effects of miR-185 overexpression on cellular autophagy and apoptosis were investigated. In order to explore the molecular mechanisms underlying the effects of miR-185 during PD pathogenesis, the 5'-adenosine monophosphate-activated protein kinase (AMPK)/mechanistic target of rapamycin (mTOR) signaling pathway was examined. The aim of the present study was to investigate whether the aberrant expression of miR-185 played a key role in the pathogenesis of PDFindings of this study may provide a basis for the development of new therapeutic approaches of this disease.

\section{Materials and methods}

Cell culture. The human SH-SY5Y DAergic neuroblastoma cell line was purchased from American Type Culture Collection (Manassas, VA, USA). Cells were cultured in Dulbecco's modified Eagle's medium, supplemented with $10 \%$ fetal bovine serum (Gibco; Thermo Fisher Scientific, Inc., Waltham, MA, USA) and $100 \mathrm{U} / \mathrm{ml}$ penicillin (Sigma-Aldrich; Merck KGaA, Darmstadt, Germany), and incubated at $37^{\circ} \mathrm{C}$ in a humidified $5 \% \mathrm{CO}_{2}$ atmosphere.

MPTP has been reported to produce irreversible and severe Parkinsonism, and has widely been used for the establishment of PD models (19). 1-Methyl-4-phenylpyridinium is the active metabolite of MPTP, and is a potent neurotoxin for SH-SY5Y cells and DAergic neurons in the substantia nigra (20). In the present study, SH-SY5Y cells were treated with 0, 50, 100, 200 or $400 \mu \mathrm{M}$ MPTP (Sigma-Aldrich; Merck KGaA) for $24 \mathrm{~h}$ to generate an in vitro PD model. SH-SY5Y cells were then harvested for further analysis.

Cell transfection. For SH-SY5Y cell transfection, a miR-185 mimic and scramble miRNA were purchased from Sangon Biotech Co., Ltd. (Shanghai, China). The miR-185 mimic sequence was 5'-UGGAGAGAAAGGCAGUUCCUGA-3' and the scramble control sequence was 5'-UUGUACUACACA AAAGUACUG-3'. miRNAs were transfected into SH-SY5Y cells $\left(1 \times 10^{5}\right.$ cells/well) using Lipofectamine ${ }^{\circledR} 2000$ reagent (Invitrogen; Thermo Fisher Scientific, Inc.) according to the manufacturer's protocol. Cells transfected with scramble miRNA were used as the negative control. Cells were maintained in Neurobasal medium (Invitrogen; Thermo Fisher Scientific, Inc.) with 2\% B27 (Sigma-Aldrich; Merck KGaA) following transfection with miR-185 or scramble controls for $24 \mathrm{~h}$. Following $24 \mathrm{~h}$ of transfection, the SH-SY5Y cells were incubated with or without AMPK inhibitor Compound C (2 $\mu \mathrm{M}$; Calbiochem; EMD Millipore, Billerica, MD, USA) in the presence or absence of MPTP.

Cell apoptosis. An Annexin V-fluorescein isothiocyanate (FITC) cell apoptosis kit (cat. no. V13241; Invitrogen; Thermo Fisher Scientific, Inc.) was used to assess SH-SY5Y cell apoptosis by flow cytometry. Briefly, following transfection with miR-185 and scramble miRNAs for $48 \mathrm{~h}$, cells were harvested by trypsinization and resuspended in the staining buffer. Cells were fixed with $4 \%$ formaldehyde for $30 \mathrm{~min}$ at room temperature and permeabilized with $0.2 \%$ Triton X-100 in PBS for $5 \mathrm{~min}$ at room temperature and were incubated for $10 \mathrm{~min}$ with $5 \mu \mathrm{l}$ Annexin-V-FITC and $5 \mu \mathrm{l}$ propidium iodide (PI) at room temperature $\left(22^{\circ} \mathrm{C}\right)$. Apoptotic cells, identified as Annexin $\mathrm{V}$-positive and PI-negative cells, were subsequently analyzed by flow cytometry using CellQuest software version 5.2 (BD Biosciences, San Jose, CA, USA).

Reverse transcription-quantitative polymerase chain reaction (RT-qPCR). Following transfection with miR-185 or scramble controls for $24 \mathrm{~h}$, total RNA was extracted from cells ( $2 \times 10^{6}$ cells) using TRIzol ${ }^{\circledR}$ reagent (Invitrogen; Thermo Fisher Scientific, Inc.), according to the manufacturer's protocol. Total RNA was reverse transcribed into cDNA using the iScript ${ }^{\mathrm{TM}}$ cDNA Synthesis kit (Bio-Rad Laboratories, Inc., Hercules, CA, USA), according to the manufacturer's protocol. qPCR was performed using SYBR-Green Master mix (Thermo Fisher Scientific, Inc.). Primers used for qPCR amplification are presented in Table I, and GAPDH was used as the internal control. Following heating to $94^{\circ} \mathrm{C}$ for $2 \mathrm{~min}$, the experimental reaction with a volume of $50 \mu \mathrm{l}$ was subjected to 32 cycles of $94^{\circ} \mathrm{C}$ for $30 \mathrm{sec}, 61^{\circ} \mathrm{C}$ for $30 \mathrm{sec}$, and $72^{\circ} \mathrm{C}$ for $30 \mathrm{sec}$, and the PCR products were analyzed by $1.5 \%$ gel electrophoresis. Relative gene expression was calculated using the $2^{-\Delta \Delta C q}$ method (21).

Western blot analysis. Cells were washed twice with ice-cold PBS, collected and lysed using $2 \mathrm{ml}$ lysis buffer. Following transfection with miR-185 or scramble controls for $24 \mathrm{~h}$, total protein was extracted from cells $\left(2 \times 10^{6}\right.$ cells) using radioimmunoprecipitation assay lysis buffer (50 mM Tris, $150 \mathrm{mM} \mathrm{NaCl}, 2 \mathrm{mM}$ EDTA, $1 \%$ Triton X-100, 0.1\% sodium dodecyl sulfate; $\mathrm{pH} 7.8$; Sangon Biotech Co., Ltd.). The lysates were centrifuged at 10,000 x g for $10 \mathrm{~min}$ at $4^{\circ} \mathrm{C}$. Protein concentration was detected using the bicinchoninic acid Protein Assay kit (Pierce; Thermo Fisher Scientific, Inc.). Equal quantities of extracted protein samples $(\sim 50 \mu \mathrm{g})$ were separated by $10 \%$ SDS-PAGE and transferred onto polyvinylidene difluoride membranes. Membranes were blocked with phosphate-buffered saline containing $0.2 \%$ Tween-20 and $5 \%$ non-fat milk at $4^{\circ} \mathrm{C}$ overnight, and then incubated with the following primary antibodies overnight at $4^{\circ} \mathrm{C}$ : Anti-Beclin 1 (cat. 
Table I. Primer sequences used for reverse transcription-quantitative polymerase chain reaction.

\begin{tabular}{lll}
\hline Gene & \multicolumn{1}{c}{ Forward primer $\left(5^{\prime}-3^{\prime}\right)$} & \multicolumn{1}{c}{ Reverse primer $\left(5^{\prime}-3^{\prime}\right)$} \\
\hline miR-185 & GGTGGAGAGAAAGGCAGT & TGCGTGTCGTGGAGTC \\
Beclin 1 & CAGAGCGATGGTACCTCTCTGGAGGCCTCG & CTGACCAGGGCTGGCAACTCTAGATGGC \\
LC3I & TCCGACCGGCCTTTCAAGCAG & GAGAACCTGACCAGAACTCCCAG \\
LC3II & AAACGCATTTGCCATCACAGT & GTGAGGACTTTGGGTGTGGTTC \\
AMPK & GCTGTGGATCGCCAAATTAT & CACGTGCTCATCATCGAAAG \\
mTOR & ATTTGATCAGGTGTGCCAGT & GCTTAGGACATGGTTCATGG \\
GAPDH & GGGAGCCAAAAGGGTCAT & GAGTCCTTCCACGATACCAA
\end{tabular}

miR, microRNA; AMPK, 5'-adenosine monophosphate-activated protein kinase; mTOR, mechanistic target of rapamycin.

no. sc-48341; 1:1,000; Santa Cruz Biotechnology, Inc. Dallas, TX, USA), anti-microtubule-associated protein light chain 3 (LC3)I (cat. no. sc-16756; 1:1,000; Santa Cruz Biotechnology, Inc.), anti-LC3II, anti-AMPK (cat. no. ab80039; 1:1,000; Abcam, Cambridge, UK), anti-p-AMPK (cat. no. ab133448; 1:1,000; Abcam), anti-mTOR (cat. no. sc-293089; 1:1,000; Santa Cruz Biotechnology, Inc.), anti-p-mTOR (cat. no. sc-293133; 1:1,000; Santa Cruz Biotechnology, Inc.), and anti-GAPDH (cat. no. sc-293335; 1:5,000; Santa Cruz Biotechnology, Inc.). Membranes were then probed with horseradish peroxidase-conjugated secondary antibodies (cat. nos. ab131368 and ab191866; both 1:5,000; Abcam) for $2 \mathrm{~h}$ at room temperature. Protein bands were visualized by enhanced chemiluminescence (ECL) using an ECL Western Blotting Detection system (GE Healthcare, Chicago, IL, USA). GAPDH was used as the internal control.

Statistical analysis. Differences among groups were assessed using one-way analysis of variance followed by the least significant difference test. Data are expressed as the mean \pm standard deviation of at least three independent experiments. Statistical analysis was performed using GraphPad Prism software (version 5.0; GraphPad Software, Inc., La Jolla, CA, USA). P<0.05 was considered to indicate a statistically significant difference.

\section{Results}

miR-185 is downregulated in MPTP-treated SH-SY5Y cells. In the present study, RT-qPCR was used to assess the expression of miR-185 in MPTP-treated SH-SY5Y cells. As shown in Fig. 1, the expression levels of miR-185 in SH-SY5Y cells exhibited a dose-dependent decrease following treatment with increasing MPTP concentrations. Notably, miR-185 expression was significantly downregulated following treatment with MPTP at concentrations of $>100 \mu \mathrm{M}$ when compared with untreated controls $(100 \mu \mathrm{M}, \mathrm{P}<0.05 ; 200 \mu \mathrm{M}, \mathrm{P}<0.01 ; 400 \mu \mathrm{M}$, $\mathrm{P}<0.001)$. These results suggest that miR-185 may be downregulated in DAergic neurons during the pathogenesis of PD.

miR-185 overexpression reduces MPTP-induced apoptosis of SH-SY5Y cells. In order to investigate the effects of miR-185 in MPTP-induced PD, SH-SY5Y cells were transfected with a miR-185 mimic or a negative control. As shown in Fig. 2A, the expression of miR-185 in SH-SY5Y cells was significantly increased following transfection with the

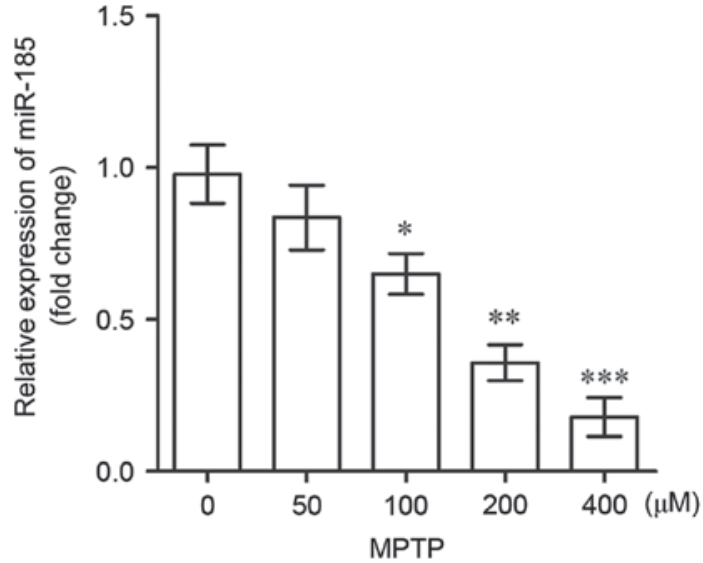

Figure 1. miR-185 expression levels in human SH-SY5Y dopaminergic neuroblastoma cells following treatment with $0,50,100,200$ and $400 \mu \mathrm{M}$ MPTP, as determined by reverse transcription-quantitative polymerase chain reaction. MPTP at concentrations $>100 \mu \mathrm{M}$ significantly downregulated the expression of miR-185. Data are expressed as the mean \pm standard deviation. ${ }^{*} \mathrm{P}<0.05,{ }^{* * *} \mathrm{P}<0.01,{ }^{* * * *} \mathrm{P}<0.001$ vs. $0 \mu \mathrm{M}$ MPTP. miR, microRNA; MPTP, 1-methyl-4-phenyl-1,2,3,6-tetrahydropyridine.

miR-185 mimic when compared with untransfected control cells $(\mathrm{P}<0.01)$, indicating that miR-185 was successfully overexpressed in MPTP-treated SH-SY5Y cells. In addition, flow cytometric analysis revealed that treatment with MPTP was associated with a significant increase in the percentage of apoptotic SH-SY5Y cells, whereas miR-185 overexpression significantly suppressed MPTP-induced cell apoptosis $(\mathrm{P}<0.05$; Fig. 2B and C).

miR-185 overexpression decreases MPTP-induced autophagy of SH-SY5Y cells. The effects of miR-185 overexpression on MPTP-induced SH-SY5Y cell autophagy were investigated by assessing the protein expression of the autophagy markers, Beclin 1, LC3I and LC3II. As shown in Fig. 3, the mRNA expression levels of Beclin 1 and the LC3II/LC3I ratio were significantly upregulated in SH-SY5Y cells following treatment with MPTP. Notably, miR-185 overexpression significantly suppressed the MPTP-induced upregulation in the expression levels of these autophagy markers $(\mathrm{P}<0.05)$. Similar results were identified in the western blot analysis. These results suggested that miR-185 overexpression may decrease MPTP-induced cell autophagy. 

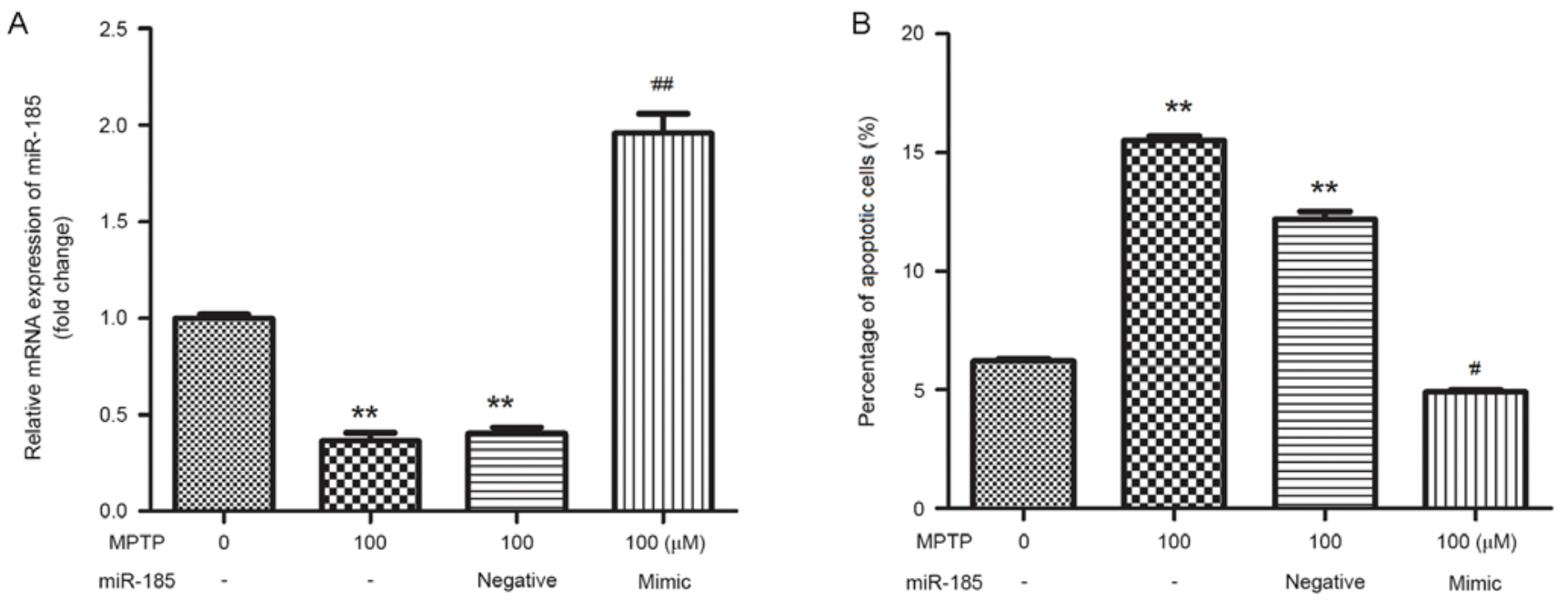

C

Annexin V-FITC
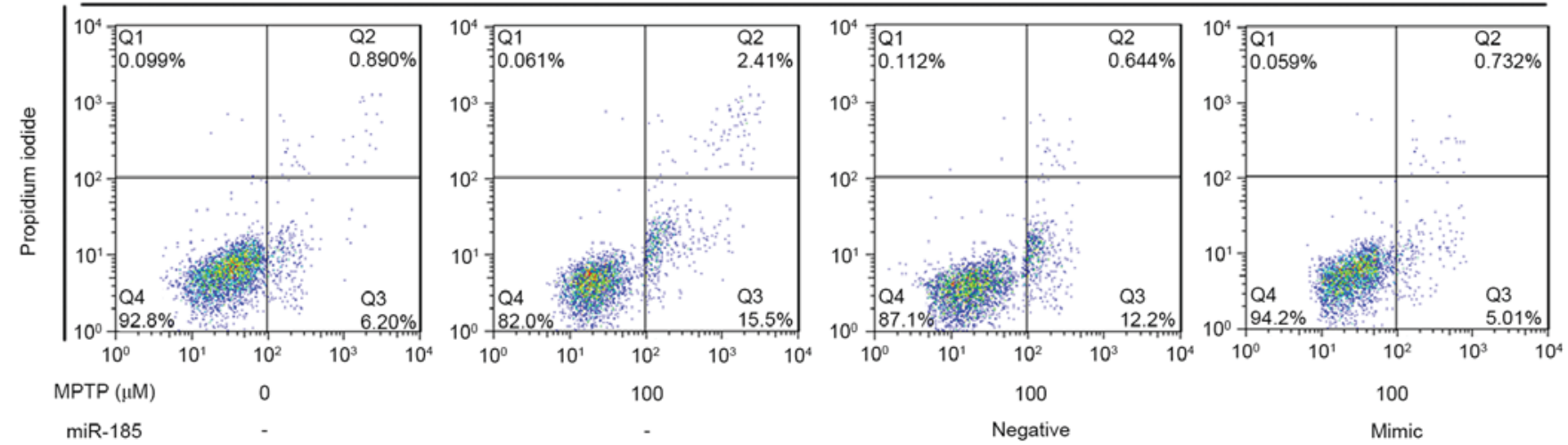

Figure 2. miR-185 overexpression prevented MPTP-induced apoptosis in human SH-SY5Y dopaminergic neuroblastoma cells. (A) miR-185 expression levels in MPTP-treated SH-SY5Y cells following transfection with a miR-185 mimic or negative control miRNA were assessed using reverse transcription-quantitative polymerase chain reaction. (B) The percentage of apoptotic cells was significantly decreased following miR-185 overexpression in MPTP-treated SH-SY5Y cells. (C) Flow cytometry was employed to assess cellular apoptosis following treatment with MPTP and transfection with miR-185 mimic or scramble miRNA. Control cells received no treatment with MPTP and were not transfected. Data are expressed as the mean \pm standard deviation. ${ }^{*} \mathrm{P}<0.05$ and ${ }^{* *} \mathrm{P}<0.01$ vs. untreated control; ${ }^{\#} \mathrm{P}<0.05$ and ${ }^{\# \#} \mathrm{P}<0.01$ vs. MPTP-treated negative control. miR, microRNA; MPTP, 1-methyl-4-phenyl-1,2,3,6-tetrahydropyridine; FITC, fluorescein isothiocyanate; negative, scramble miRNA.

miR-185 overexpression suppresses activation of the AMPK/mTOR signaling pathway. To further investigate the molecular mechanisms underlying the involvement of miR-185 in PD pathogenesis, the AMPK/mTOR signaling pathway was examined. As shown in Fig. 4A, miR-185 overexpression was revealed to significantly inhibit the MPTP-enhanced upregulation of AMPK and mTOR mRNA $(\mathrm{P}<0.05)$, which suggests that miR-185 overexpression suppresses activation of the AMPK/mTOR signaling pathway. As presented in Fig. 4B, the protein expression levels of AMPK and mTOR were not altered by MPTP or MPTP+miR-185 treatment. In addition, the MPTP-induced upregulation of p-AMPK was reduced by miR-185 transfection, whereas p-mTOR expression was increased following MPTP+miR-185 treatment. These results suggested that miR-185 did not inhibit activation of the AMPK/mTOR signaling pathway.

To further investigate the effects of miR-185 on the AMPK/mTOR signaling pathway, cells were treated with the AMPK inhibitor, Compound $\mathrm{C}$, for $1 \mathrm{~h}$, and the effects of AMPK inhibition on SH-SY5Y cell apoptosis were assessed by flow cytometry. The percentage of apoptotic SH-SY5Y cells was significantly increased following treatment with MPTP and Compound $\mathrm{C}$ when compared with cells treated with Compound $\mathrm{C}$ alone ( $\mathrm{P}<0.01$; Fig. 5). However, miR-185 overexpression significantly suppressed SH-SY5Y cell apoptosis following co-treatment with MPTP and Compound C compared with the MPTP+Compound C+blank and MPTP+Compound $\mathrm{C}+$ negative groups $(\mathrm{P}<0.05)$. These results suggest that miR-185 may be involved in the regulation of MPTP-induced apoptosis via modulation of the AMPK/mTOR signaling pathway.

\section{Discussion}

In the present study, the role of miR-185 in the regulation of cellular autophagy and apoptosis were investigated in an MPTP-induced in vitro model of PD, and the underlying molecular mechanisms were explored. The results demonstrated that miR-185 was significantly downregulated in MPTP-treated SH-SY5Y DAergic cells. Notably, miR-185 overexpression was revealed to significantly suppress MPTP-induced apoptosis and autophagy in SH-SY5Y cells. In addition, miR-185 
A

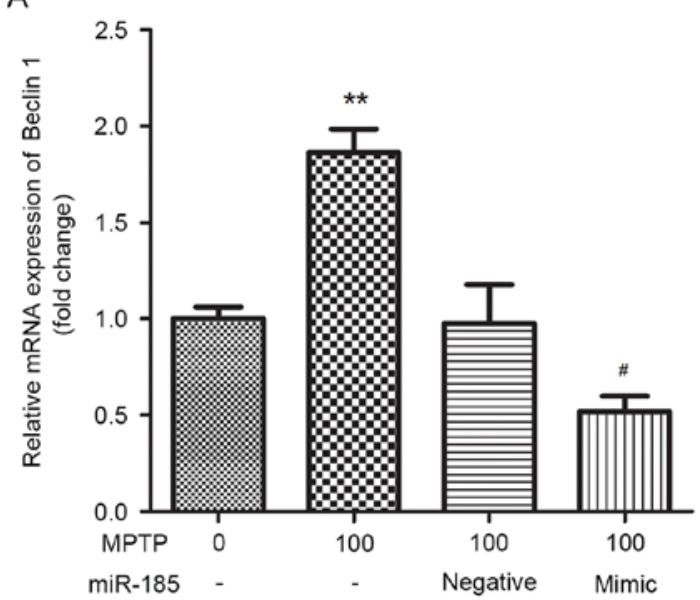

C

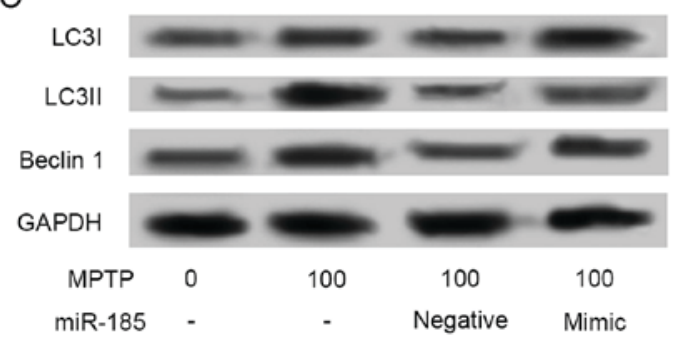

B

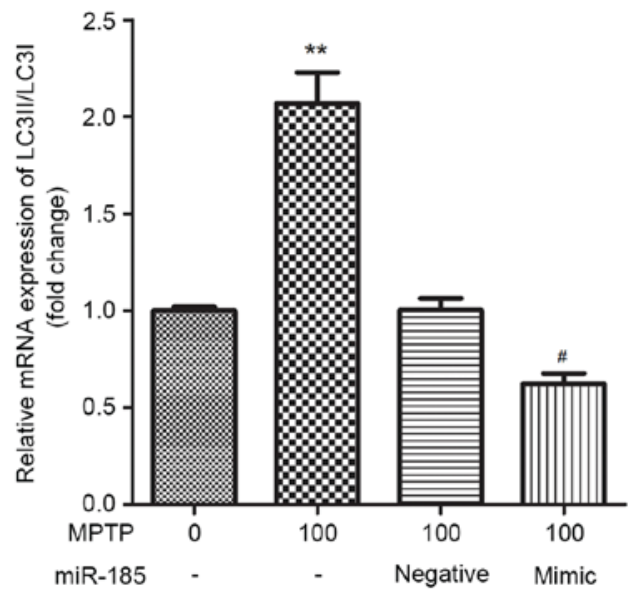

Figure 3. miR-185 overexpression suppressed MPTP-induced autophagy in human SH-SY5Y dopaminergic neuroblastoma cells. The mRNA expression levels of the autophagy markers (A) Beclin 1 and (B) LC3II/LC3I were assessed in MPTP-treated SH-SY5Y cells following transfection with a miR-185 mimic or negative control miRNA using reverse transcription-quantitative polymerase chain reaction. miR-185 overexpression significantly downregulated the mRNA expression levels of Beclin 1 and the LC3II/LC3I ratio in MPTP-treated SH-SY5Y cells. Data are expressed as the mean \pm standard deviation. ${ }^{* *} \mathrm{P}<0.01$ vs. untreated control; " $\mathrm{P}<0.05$ vs. MPTP-treated negative control. (C) The protein expression levels of Beclin 1, LC3I and LC3II were assessed by western blot analysis. Control cells received no treatment with MPTP and were not transfected. miR, microRNA; MPTP, 1-methyl-4-phenyl-1,2,3,6-tetrahydropyridine; LC3, microtubule-associated protein light chain 3; negative, scramble miRNA.

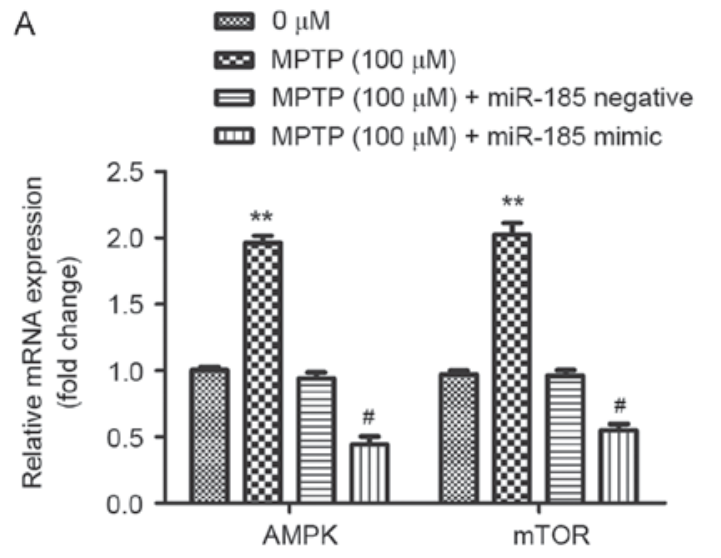

B

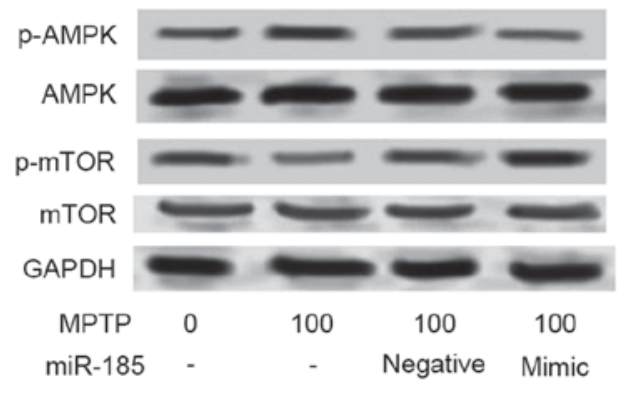

Figure 4. miR-185 overexpression suppressed MPTP-induced activation of the AMPK/mTOR signaling pathway in human SH-SY5Y dopaminergic neuroblastoma cells. (A) The mRNA expression levels of AMPK and mTOR were assessed in MPTP-treated SH-SY5Y cells following transfection with an miR-185 mimic or negative control miRNA using reverse transcription-quantitative polymerase chain reaction. miR-185 overexpression significantly downregulated the mRNA expression levels of AMPK and mTOR in MPTP-treated SH-SY5Y cells. Data are expressed as the mean \pm standard deviation. ** P $<0.01$ vs. untreated control; " $\mathrm{P}<0.05$ vs. MPTP-treated negative control. (B) The protein expression levels of AMPK, $\mathrm{p}$-AMPK, mTOR and p-mTOR were assessed by western blot analysis. Control cells received no treatment with MPTP and were not transfected. miR, microRNA; MPTP, 1-methyl-4-phenyl-1,2,3,6-tetrahydropyridine; AMPK, 5'-adenosine monophosphate-activated protein kinase; mTOR, mechanistic target of rapamycin; p-, phosphorylated; negative, scramble miRNA.

overexpression was demonstrated to inhibit activation of the AMPK/mTOR signaling pathway in MPTP-treated cells. These results suggest that miR-185 may be involved in the pathogenesis and progression of PD.
Apoptosis and autophagy are basic cellular processes, which are essential for the maintenance of neuronal homeostasis under physiological conditions. Dysfunction of these processes has been reported in various neurodegenerative diseases, including 


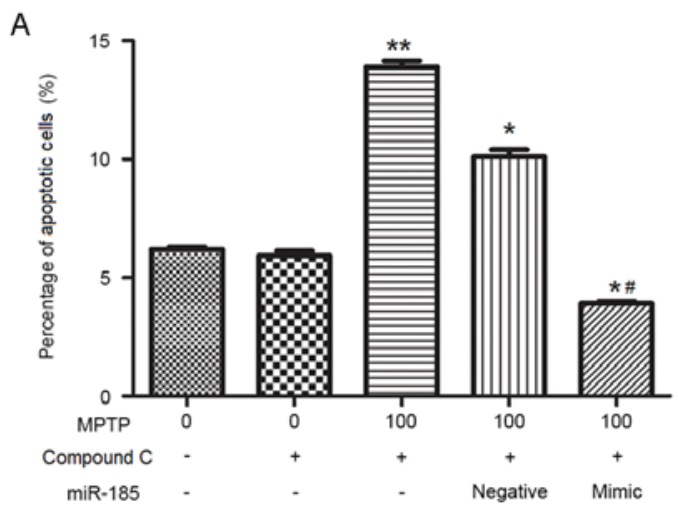

B
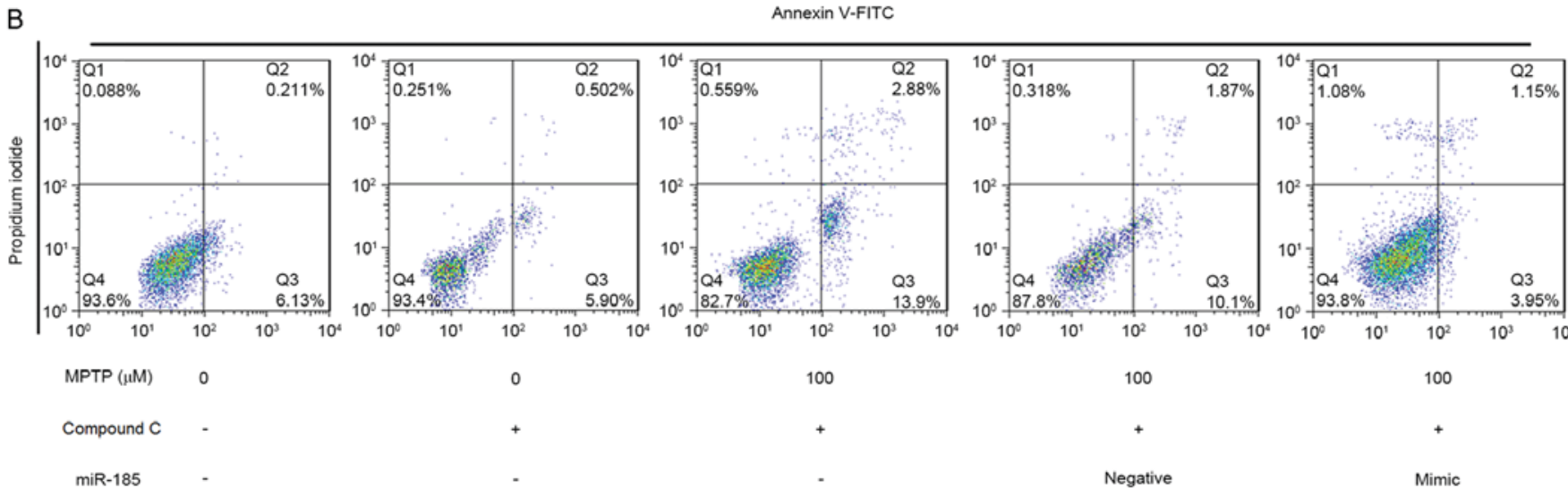

100

100

100

$+$

Negative

$+$

Mimic

Figure 5. miR-185 overexpression inhibited MPTP-induced apoptosis in human SH-SY5Y dopaminergic neuroblastoma cells following treatment with the AMPK inhibitor Compound C. (A) The percentage of apoptotic cells was significantly decreased following miR-185 overexpression in MPTP- and Compound C-treated SH-SY5Y cells. (B) Flow cytometry was used to assess cellular apoptosis following treatment with MPTP and Compound C, and transfection with miR-185 mimic or negative control miRNA. Control cells received no treatment with MPTP or Compound C, and were not transfected. Data are expressed as the mean \pm standard deviation. ${ }^{*} \mathrm{P}<0.05$ and ${ }^{* *} \mathrm{P}<0.01$ vs. untreated control; ${ }^{*} \mathrm{P}<0.05$ vs. MPTP-treated negative control. miR, microRNA; MPTP, 1-methyl-4-phenyl-1,2,3,6-tetrahydropyridine; AMPK, 5'-adenosine monophosphate-activated protein kinase; FITC, fluorescein isothiocyanate; negative, scramble miRNA.

PD (22-24). Apoptosis has been previously identified as a critical factor that contributes to neuronal degradation in PD (25). In addition, dysregulation of autophagy processes has been reported in animal models of PD, as well as in samples isolated from patients with PD (26). Beclin 1 upregulation has been revealed to prevent apoptosis and enhance the activity of autophagy signaling pathways (26). An age-dependent downregulation in Beclin 1 expression has been demonstrated to occur in the brain, which has been associated with a reduction in autophagy during the progression of neurodegenerative diseases (27).

miR-185 has been identified as a crucial regulator of cell death processes in lung epithelial cells induced by oxidative stress (28). In addition, miR-185-3p has been reported to modulate the growth and apoptosis of nasopharyngeal carcinoma cells (29). In the present study, miR-185 overexpression was revealed to significantly suppress MPTP-induced apoptosis of SH-SY5Y cells. Furthermore, miR-185 overexpression significantly downregulated the expression of the autophagy markers, Beclin 1 and the LC3II/LC3I ratio, in MPTP-treated cells, thus indicating suppression of cell autophagy. The LC3II/LC3I ratio is a well-established biochemical assay for the activation of autophagy (30). These results are in accordance with previous studies $(22,23)$ suggesting that miR-185 may be implicated in the progression of $\mathrm{PD}$ via the regulation of cellular apoptosis and autophagy signaling pathways.
To further investigate the molecular mechanisms underlying the involvement of miR-185 in the pathogenesis of $\mathrm{PD}$, the effects of $\mathrm{miR}-185$ on the AMPK/mTOR signaling pathway were examined. mTOR has been identified as an indirect target of AMPK, and is negatively regulated by AMPK; a process that is critical for the regulation of cell growth and survival $(31,32)$. In addition, mTOR has been demonstrated to induce apoptosis and autophagy during the development of neurocardiac complications in diabetes mellitus (33), and AMPK-induced mTOR inhibition reportedly occurs in autophagy processes during neuronal cell death induced by the neurotoxin, tributyltin (34). Hydrogen peroxide has been revealed to inhibit mTOR signaling and lead to neuronal apoptosis through the activation of AMPK (35). In addition, Arsikin et al (36) demonstrated that AMPK/mTOR-dependent autophagy contributed to the induction of oxidative stress, thus leading to SH-SY5Y cell apoptosis. In the present study, miR-185 overexpression downregulated the mRNA expression of AMPK and mTOR, which had been enhanced following treatment with MPTP. Furthermore, miR-185 overexpression significantly suppressed apoptosis of SH-SY5Y cells treated with MPTP and an AMPK inhibitor simultaneously. These results suggested that miR-185 overexpression may suppress the death of DAergic neurons in the substantia nigra, and therefore prevent the development and progression of PD via 
inhibition of the AMPK/mTOR signaling pathway. However, further studies are required to fully elucidate the association between miR-185 and the AMPK/mTOR signaling pathway, and explore the molecular mechanisms underlying their roles in the pathogenesis of PD.

In conclusion, the results of the present study suggest that miR-185 may inhibit neuronal autophagy and apoptosis via regulation of the $\mathrm{AMPK} / \mathrm{mTOR}$ intracellular signaling pathway in PD. Therefore, the authors hypothesize that AMPK/mTOR-mediated autophagy and apoptotic signaling pathways may be potential novel therapeutic targets for the development of alternative strategies for the treatment of patients with PD.

\section{Acknowledgements}

The present study was supported by the National Nature Science Foundation of China (grant nos. 81171315 and 81227902-5).

\section{References}

1. Moore DJ, West AB, Dawson VL and Dawson TM: Molecular pathophysiology of Parkinson's disease. Annu Rev Neurosci 28 57-87, 2005.

2. Valente EM, Abou-Sleiman PM, Caputo V, Muqit MM, Harvey K, Gispert S, Ali Z, Del Turco D, Bentivoglio AR, Healy DG, et al: Hereditary early-onset Parkinson's disease caused by mutations in PINK1. Science 304: 1158-1160, 2004.

3. Dexter DT and Jenner P: Parkinson disease: From pathology to molecular disease mechanisms. Free Radic Biol Med 62: 132-144, 2013

4. Olanow CW and Tatton WG: Etiology and pathogenesis of Parkinson's disease. Annu Rev Neurosci 22: 123-144, 1999.

5. Jankovic J: Parkinson's disease: Clinical features and diagnosis. J Neurol Neurosurg Psychiatry 79: 368-376, 2008

6. Bonifati V: Genetics of Parkinson's disease-state of the art, 2013. Parkinsonism Relat Disord 20 (Suppl 1): S23-S28, 2014.

7. Campdelacreu J: Parkinson disease and Alzheimer disease: Environmental risk factors. Neurologia 29: 541-549, 2014 (In English, Spanish).

8. Collier TJ, Kanaan NM and Kordower JH: Ageing as a primary risk factor for Parkinson's disease: Evidence from studies of non-human primates. Nat Rev Neurosci 12: 359-366, 2011.

9. Hwang O: Role of oxidative stress in Parkinson's disease. Exp Neurobiol 22: 11-17, 2013.

10. Noyce AJ, Bestwick JP, Silveira-Moriyama L, Hawkes CH, Giovannoni G, Lees AJ and Schrag A: Meta-analysis of early nonmotor features and risk factors for Parkinson disease. Ann Neurol 72: 893-901, 2012.

11. Mouradian MM: MicroRNAs in Parkinson's disease. Neurobiol Dis 46: 279-284, 2012.

12. Petillo D, Orey S, Tan AC, Forsgren L and Khoo SK: Parkinson's disease-related circulating microRNA biomarkers-a validation study. AIMS Med Sci 2: 7-14, 2015.

13. Khoo SK, Petillo D, Kang UJ, Resau JH, Berryhill B, Linder J, Forsgren L, Neuman LA and Tan AC: Plasma-based circulating microRNA biomarkers for Parkinson's disease. J Parkinsons Dis 2: 321-331, 2012.

14. Hao B, Chen X, Dai D, Zou C, Wu X and Chen J: Bioinformatic analysis of microRNA expression in Parkinson's disease. Mol Med Rep 11: 1079-1084, 2015.

15. Wang H, Ye Y, Zhu Z, Mo L, Lin C, Wang Q, Wang H, Gong X, He X, Lu G, et al: MiR-124 regulates apoptosis and autophagy process in MPTP model of Parkinson's disease by targeting to bim. Brain Pathol 26: 167-176, 2016.

16. Li S, Lv X, Zhai K, Xu R, Zhang Y, Zhao S, Qin X, Yin L and Lou J: MicroRNA-7 inhibits neuronal apoptosis in a cellular Parkinson's disease model by targeting Bax and Sirt2. Am J Transl Res 8: 993-1004, 2016.
17. Zhou Y, Lu M, Du RH, Qiao C, Jiang CY, Zhang KZ, Ding JH and Hu G: MicroRNA-7 targets Nod-like receptor protein 3 inflammasome to modulate neuroinflammation in the pathogenesis of Parkinson's disease. Mol Neurodegener 11: 28, 2016.

18. Ding H, Huang Z, Chen M, Wang C, Chen X, Chen J and Zhang J: Identification of a panel of five serum miRNAs as a biomarker for Parkinson's disease. Parkinsonism Relat Disord 22: 68-73, 2016.

19. Langston JW and Irwin I: MPTP: Current concepts and controversies. Clin Neuropharmacol 9: 485-507, 1986.

20. Itano $Y$ and Nomura Y: 1-Methyl-4-phenyl-pyridinium ion $(\mathrm{MPP}+)$ causes DNA fragmentation and increases the Bcl-2 expression in human neuroblastoma, SH-SY5Y cells, through different mechanisms. Brain Res 704: 240-245, 1995.

21. Livak KJ and Schmittgen TD: Analysis of relative gene expression data using real-time quantitative PCR and the 2(-Delta Delta C(T)) method. Methods 25: 402-408, 2001.

22. Ghavami S, Shojaei S, Yeganeh B, Ande SR, Jangamreddy JR, Mehrpour M, Christoffersson J, Chaabane W, Moghadam AR, Kashani HH, et al: Autophagy and apoptosis dysfunction in neurodegenerative disorders. Prog Neurobiol 112: 24-49, 2014.

23. Xiong N, Xiong J, Jia M, Liu L, Zhang X, Chen Z, Huang J, Zhang Z, Hou L, Luo Z, et al: The role of autophagy in Parkinson's disease: Rotenone-based modeling. Behav Brain Funct 9: 13, 2013.

24. Perier C, Bové J and Vila M: Mitochondria and programmed cell death in Parkinson's disease: Apoptosis and beyond. Antioxid Redox Signal 16: 883-895, 2012.

25. Tatton WG, Chalmers-Redman R, Brown D and Tatton N: Apoptosis in Parkinson's disease: Signals for neuronal degradation. Ann Neurol 53 (Suppl 3): S61-S72, 2003.

26. Lynch-Day MA, Mao K, Wang K, Zhao M and Klionsky DJ: The role of autophagy in Parkinson's disease. Cold Spring Harb Perspect Med 2: a009357, 2012.

27. Pickford F, Masliah E, Britschgi M, Lucin K, Narasimhan R, Jaeger PA, Small S, Spencer B, Rockenstein E, Levine B and Wyss-Coray T: The autophagy-related protein beclin 1 shows reduced expression in early Alzheimer disease and regulates amyloid beta accumulation in mice. J Clin Invest 118: 2190-2199, 2008.

28. Zhang D, Lee H, Cao Y, Dela Cruz CS and Jin Y: MiR-185 mediates lung epithelial cell death after oxidative stress. Am J Physiol Lung Cell Mol Physiol 310: L700-L710, 2016.

29. Xu J, Ai Q, Cao H and Liu Q: MiR-185-3p and miR-324-3p predict radiosensitivity of nasopharyngeal carcinoma and modulate cancer cell growth and apoptosis by targeting SMAD7. Med Sci Monit 21: 2828-2836, 2015.

30. Mizushima N, Yamamoto A, Matsui M, Yoshimori T and Ohsumi Y: In vivo analysis of autophagy in response to nutrient starvation using transgenic mice expressing a fluorescent autophagosome marker. Mol Biol Cell 15: 1101-1111, 2004.

31. Schmelzle T and Hall MN: TOR, a central controller of cell growth. Cell 103: 253-262, 2000.

32. Hay N and Sonenberg N: Upstream and downstream of mTOR. Genes Dev 18: 1926-1945, 2004.

33. Maiese K: mTOR: Driving apoptosis and autophagy for neurocardiac complications of diabetes mellitus. World J Diabetes 6: 217-224, 2015.

34. Nakatsu Y, Kotake Y, Takai N and Ohta S: Involvement of autophagy via mammalian target of rapamycin (mTOR) inhibition in tributyltin-induced neuronal cell death. J Toxicol Sci 35: 245-251,2010.

35. Chen L, Xu B, Liu L, Luo Y, Yin J, Zhou H, Chen W, Shen T, Han X and Huang S: Hydrogen peroxide inhibits mTOR signaling by activation of AMPKalpha leading to apoptosis of neuronal cells. Lab Invest 90: 762-773, 2010.

36. Arsikin K, Kravic-Stevovic T, Jovanovic M, Ristic B, Tovilovic G, Zogovic N, Bumbasirevic V, Trajkovic V and Harhaji-Trajkovic L: Autophagy-dependent and-independent involvement of AMPactivated protein kinase in 6-hydroxydopamine toxicity to SH-SY5Y neuroblastoma cells. Biochim Biophys Acta 1822: 1826-1836, 2012

This work is licensed under a Creative Commons Attribution-NonCommercial-NoDerivatives 4.0 International (CC BY-NC-ND 4.0) License. 